Presentazione a Gisella Gaspari e Matteo Pasetti, «Il Pci è un partito finito». Video-documentario sulla scomparsa della "piazza rossa"
ISBN: 978-88-98392-05-6 DOI: 10.12977/ereview4 pp. $145-151$

Attraverso immagini di manifestazioni a sfondo politico nelle piazze di Bologna, il videodocumentario illustra una trasformazione delle forme di partecipazione collettiva alla vita pubblica, che negli anni Settanta ha visto un ridimensionamento del ruolo dei partiti di massa, e in particolare del Pci.

Through motion pictures about political demonstrations in Bologna's squares, this videodocumentary illustrates a trasformation of forms of collective participation in public life, which in the 70's saw a reduction in the role of the mass parties, especially the Italian Communist Party.

\title{
1. Presentazione
}

Il video-documentario (䀠 $\mathrm{http}: / /$ youtu.be/N_mJEV7sOsw) che presentiamo intende fornire un piccolo contributo - di tipo impressionistico, si potrebbe dire - al dossier sulla crisi dei partiti in Emilia Romagna tra gli anni Settanta e Ottanta, attraverso una selezione di immagini che mostrano alcuni frammenti di vita politica nelle piazze bolognesi. L'idea di fondo, che si vuole suggerire visivamente, è che la crisi si manifestò anche come trasformazione delle forme di partecipazione collettiva alla vita pubblica, con un progressivo ridimensionamento del ruolo dei grandi partiti di massa (e in particolare del partito comunista, che nel capoluogo e in gran parte della regione deteneva una netta maggioranza elettorale, e quindi la guida di molte amministrazioni comunali, di tutte quelle provinciali e dell'ente 
regionale). Si tratta di un aspetto ampiamente sottolineato dalle analisi politologiche, sociologiche o storiografiche sul tema, ma che un racconto per immagini può aiutare a mettere a fuoco.

\section{Iniziando dalla fine}

Il filmato si apre con un ragazzo che prende la parola sul palco di una manifestazione in piazza Verdi, nella zona universitaria di Bologna, il $1^{\circ}$ giugno 1980. Lo si vede dichiarare la propria appartenenza alla frangia dei "nichilisti", trattenere a stento una risata d'imbarazzo, poi andare subito all'attacco:

Noi siamo per la linea dura, per l'opposizione totale al comune, perché pensiamo che il comune sia una grossa macchina di integrazione giovanile, visto che il Pc, che vuol dire comune in ogni caso, anche se ci sono degli altri partiti, è un partito finito, un partito marcio, che probabilmente un domani non avrà più niente da dire.

Il giovane "nichilista" - che in seguito con il nome d'arte di Steno e il gruppo musicale Nabat si sarebbe ritagliato un posto di primo piano nella nicchia del punkrock italiano - esprimeva in questi termini il proprio dissenso per l'attesissimo concerto dei Clash, previsto per la sera stessa in piazza Maggiore. A suo modo di vedere, condiviso perlomeno dagli altri promotori dell'adunata pomeridiana contro il grande evento serale, la band britannica stava con ogni evidenza tradendo i principi professati: che cosa ci facevano in piazza Maggiore, su invito del comune di Bologna, gli esponenti più celebri e celebrati del movimento punk, quelli che cantavano London's Burning, I Fought the Law o Revolution Rock, arrivando a sfoggiare, in qualche occasione, la maglietta con la stella delle Brigate rosse? Perché questa resa di fronte alle istituzioni, questa compromissione con un partito che rappresentava "il sistema"?

Naturalmente, la parte del discorso che qui interessa non riguarda il grado di coerenza ideologica dei Clash, quanto invece l'altro bersaglio della polemica, ovvero un partito comunista totalmente incardinato nel sistema di potere, tanto da rendere lapalissiana l'identificazione con l'amministrazione comunale: il Pci (il Pci locale, ma il ragionamento poteva essere traslato, con pochi aggiustamenti, su scala nazionale) non rappresentava più la forza rivoluzionaria, il "sol dell'avvenire", ma era diventato a tutti gli effetti uno strumento di conservazione, un architrave del sistema (anzi, nel caso di Bologna e di gran parte dell'Emilia Romagna, esso coincideva con "il sistema" stesso). Secondo questa visione delle cose, del famoso ossimoro utilizzato da Berlinguer per definire il Pci, «partito di lotta e di governo», era reale solo la seconda componente: il governo, il potere. E quel che 
era peggio, si trattava ormai di un potere fine a se stesso: una «grande macchina» burocratizzata e autoritaria, volta, per esempio, non all'emancipazione ma all'«integrazione giovanile» al fine di preservare lo status quo. Di conseguenza, il partito comunista aveva rinnegato la sua natura, era «un partito finito», appunto, «un partito marcio» che non aveva «più niente da dire».

La critica al Pci espressa in quel frangente proveniva senza dubbio da una posizione minoritaria di estremismo politico e culturale. Tuttavia, al netto del ribellismo anarchico-nichilista, essa trovava corrispondenza in teorizzazioni e atteggiamenti diffusi da più di un decennio, a livello locale e nazionale, soprattutto tra le generazioni più giovani e tra le file della sinistra radicale. Anche se, a volte, in termini un po' più sofisticati, l'accusa al partito comunista (e più in generale alla forma partito in quanto tale) di funzionare come un apparato oligarchico, di essere un fattore di mera conservazione politica e sociale, di aver perduto in pratica ogni prospettiva rivoluzionaria, era presente nell'ideologia di tutti i movimenti giovanili dal Sessantotto in poi. Gli avvenimenti della seconda metà degli anni Settanta avevano allargato ulteriormente la forbice tra il Pci e la composita area della contestazione. Su scala nazionale, l'incapacità di mettere a frutto i successi elettorali del 1975-76, il "compromesso storico" e il suo fallimento, la "linea della fermezza" di fronte all'esplosione del terrorismo avevano ampliato la disillusione nei confronti del Pci come artefice del cambiamento. Su scala regionale, la lunga permanenza dei comunisti alla guida di gran parte delle amministrazioni locali aveva finito paradossalmente per accentuare l'immagine di un partito "come gli altri”. Nel capoluogo, epicentro della nuova ondata di contestazione del 1977, l'arroccamento del gruppo dirigente a difesa dell'ordine pubblico aveva chiuso ogni possibilità di dialogo col movimento studentesco, polarizzando lo scontro e trasformando il Pci in un nemico.

L'intervento posto in apertura del filmato esprimeva insomma uno stato d'animo che era meno marginale della polemica settaria contro il concerto dei Clash. D'altra parte, l'idea stessa di invitare i Clash in piazza Maggiore a spese del comune, una settimana prima delle elezioni amministrative, può essere letta come un tentativo dell'establishment politico locale per riavvicinarsi alla cultura giovanile e alle sue frange più turbolente. Solo che ormai era troppo tardi. Forse il Pci non era ancora «un partito finito» (a Bologna, alle amministrative dell'8 giugno 1980, perdendo 3 punti in percentuale rispetto al 1975, vinse pur sempre con il $46 \%$ delle preferenze). Ma la rottura con un segmento significativo delle generazioni più giovani si sarebbe rivelata irreversibile. 


\section{Un prima e un dopo}

Dopo questo prologo - che contrariamente alle norme di un buon plot cinematografico svela subito il finale, ma perché sia più intuibile il filo conduttore che tiene insieme le sequenze - il video-documentario prosegue facendo un salto indietro nel tempo, di circa trent'anni, alla prima Festa nazionale dell'Unità, tenutasi a Bologna nel settembre 1951. È da qui che si dipana il nostro racconto per immagini, che poi procede con salti cronologici via via più serrati e si conclude ellitticamente con il concerto dei Clash del giugno 1980.

Le scene coprono quindi un arco temporale (1951-1980) parzialmente sfasato rispetto al periodo preso in esame nel dossier (gli anni Settanta e Ottanta), contrapponendo due blocchi di sequenze: il primo si conclude con i festeggiamenti in piazza Maggiore per il successo elettorale del Pci alle amministrative del giugno 1975; il secondo è inerente al periodo successivo.

L'obiettivo è creare, attraverso un montaggio volutamente dicotomico, un effetto di contrasto tra un prima e un dopo, tra un certo modo di fare politica in piazza, rituale almeno fino alla prima metà degli anni Settanta, e un altro che ebbe i suoi prodromi nel decennio precedente, ma che divenne generalizzato solo in seguito. È questo, in sostanza, il tema di fondo del documentario: il passaggio tra due modalità diverse di utilizzare la piazza come scenario politico, tra due modalità diverse di manifestare. Si confronti, per esempio, l'incedere composto della marcia per la pace nel 1966 con il girotondo disordinato degli studenti nel 1978. Oppure il senso di organizzazione che si percepisce anche nello sventolio di bandiere rosse, durante i funerali delle vittime del treno Italicus, con l'improvvisazione che precede una manifestazione femminista dell'autunno 1975. È come se si passasse da una messa in scena disciplinata da una consapevole regia a una dove il regista non c'è più. E finché c'era, va da sé, il regista era il partito comunista.

Il video vorrebbe enfatizzare così - fin dal prologo e dal titolo, poi con la dicotomia tra un prima e un dopo - un'improvvisa perdita di ruolo del Pci, che da principale protagonista, promotore delle manifestazioni di piazza e punto di riferimento identitario, diventa una figura marginale, scompare dalla scena, o vi rimane solo come bersaglio della contestazione. Non si tratta, infatti, di illustrare una trasformazione esclusivamente formale, di stile, comportamenti, linguaggi; ma di evocare parimenti un cambiamento più profondo, riguardante il rapporto tra chi andava in piazza per partecipare alla vita politica e le istituzioni (in un'accezione un po' impropria del termine, che comprende anche i partiti di massa): da un certo momento in poi (per la precisione, dopo la sequenza dei festeggiamenti per l'esito delle elezioni del 1975), le immagini selezionate non esprimono più 
un consenso (verso il Pci e quello che rappresentava) ma un dissenso (contro il potere costituito, e quindi lo stesso Pci). Gli scontri del marzo 1977 tra il movimento studentesco e le forze dell'ordine rappresentano da questo punto di vista l'episodio più emblematico.

Un ulteriore aspetto che può essere sottolineato concerne la differente composizione, soprattutto anagrafica, delle piazze che compaiono nel filmato. Nel primo blocco di sequenze è evidente il carattere eterogeneo di adunate pubbliche alle quali partecipavano persone di ogni età. Nel secondo blocco, viceversa, in modo altrettanto evidente le manifestazioni presentano una spiccata omogeneità generazionale: sulla scena rimangono solo giovani ragazzi e ragazze. Viene così richiamata quella rottura tra una parte dell'universo giovanile, da un lato, partiti e istituzioni, dall'altro, che provocò la lacerazione più profonda dello scenario politico italiano di fine anni Settanta.

\section{Sulla scelta delle immagini}

Quello che presentiamo è dunque un film a tesi, in cui si sostiene che la crisi dei partiti di massa apertasi nel corso degli anni Settanta ebbe tra i suoi sintomi la propagazione di forme di partecipazione alla vita politica del tutto affrancate dagli stessi partiti, se non in aperta contrapposizione ad essi. Nel caso locale preso in considerazione, il capoluogo dell'"Emilia rossa", il fenomeno assunse forme peculiari a causa della lunga sovrapposizione di ruoli del partito comunista, da tre decenni al contempo detentore del potere amministrativo ed erede di una tradizione associativa di ispirazione rivoluzionaria fortemente radicata nel territorio. Qui, dove la "piazza rossa" aveva acquisito nel tempo la valenza di fattore identitario, la sua scomparsa - o comunque la sua perdita di rilevanza nella politica cittadina e nell'immaginario collettivo - fu più appariscente che altrove.

Le sequenze che compongono il documentario sono state selezionate in funzione di questa tesi, allo scopo di rendere visibile un nuovo uso politico della piazza, differente dal passato per almeno tre aspetti: in primo luogo, nel modo di manifestare, di stare in pubblico, di occupare gli spazi urbani; in secondo luogo, per il prevalere di sfiducia, dissenso, conflittualità verso l'intero ordine politico e sociale; infine, per una connotazione generazionale, determinata da una mobilitazione esclusivamente giovanile. Emerge così, innanzitutto, un campionario di volti, gesti, abiti, comportamenti, espressioni, slogan, diversi da un'epoca all'altra. Ed è questo probabilmente il contributo più significativo che un racconto per immagini, come quello proposto, può offrire al tema in oggetto (e in generale alla conoscenza storica del passato). Il ricorso a didascalie esplicative, in apertura di 
ogni sequenza, dovrebbe aiutare sia a contestualizzare gli episodi, sia a far emergere la loro funzionalità rispetto all'idea che si vuole esprimere.

Tranne la scena del prologo (tratta dal documentario di Angelo Rastelli, Mamma dammi la benza, realizzato per la televisione nel 2005), tutte le immagini provengono dagli archivi audiovisivi dell'Istituto Parri di Bologna e soprattutto dell'Associazione Home Movies per il recupero e la conservazione dei "film di famiglia". Sono immagini amatoriali, impresse su pellicola e recentemente digitalizzate, di qualità non sempre eccelsa (anche se uno dei criteri che ha guidato la selezione è stato la ricerca di uno standard tecnico minimo). Le riprese sono state effettuate da vari operatori, alcuni dei quali professionalmente legati al campo cinematografico (per esempio, Giampaolo Bernagozzi era docente di Cinematografia documentaria, Giorgio Zappoli un tecnico per gli impianti delle sale di proiezione), qualcuno (come Angelo Marzadori) militante del Pci, altri semplici appassionati di film amatoriali. Nello specifico, e nell'ordine di montaggio, le singole sequenze sono tratte dai seguenti fondi archivistici:

- festa dell'Unità, settembre 1951: fondo Angelo Marzadori, Archivio Home Movies;

- $\quad$ marcia per la pace, 13 marzo 1966: fondo Oreste Baldi, Archivio Home Movies;

- funerale delle vittime della strage del treno Italicus, 9 agosto 1974: fondo Giampaolo Bernagozzi, Istituto Storico Parri Emilia-Romagna;

- risultati delle elezioni amministrative e regionali, 16 giugno 1975: fondo Giampaolo Bernagozzi, Istituto Storico Parri Emilia-Romagna;

- manifestazione femminista, autunno 1975: fondo Piero Orlandi, Archivio Home Movies;

- $\quad$ scontri tra polizia e movimento studentesco, marzo 1977: fondo Cesare Ballarini, Archivio Home Movies;

- murales del movimento studentesco, estate 1977: fondo Vittorio Zappoli, Archivio Home Movies;

- $\quad$ sciopero del liceo artistico, 1978: fondo Benedetta Iandolo, Archivio Home Movies;

- comizio del Pli, anni Sessanta: fondo Enzo Donati, Archivio Home Movies.

Gran parte dei frammenti utilizzati erano privi di una traccia audio, ma abbiamo ritenuto opportuno effettuare un lavoro di sonorizzazione dell' intero video (per il quale si ringrazia Matteo Pasini), secondo un criterio il più possibile filologico. Com'è ovvio, spesso non esistono registrazioni sonore esterne degli eventi filmati, per cui l'abbinamento alle immagini di suoni, voci, musiche ha richiesto, oltre 
a una fase di ricerca documentaria, un'attenta valutazione delle opzioni a disposizione, da un lato per rendere più suggestive le immagini stesse, e dall'altro per evitare anacronismi. In alcuni casi (nello specifico: l'estratto di cinegiornale e la voce di Togliatti durante la festa dell'Unità del 1951; il discorso per il successo del Pci alle elezioni amministrative del 1975 di fronte a una piazza Maggiore gremita; le parole in sottofondo nell'ultimo comizio) l'audio inserito è apocrifo, ma crediamo che l'intervento non sia affatto scorretto da un punto di vista storiografico, e che anzi favorisca una percezione più intensa del quadro storico. In altri casi, si è preferito inserire un appropriato sottofondo musicale (una canzone per la manifestazione femminista del 1975; improvvisazioni strumentali per il movimento studentesco), oppure limitare la sonorizzazione all'aggiunta di rumori di fondo.

Tutto ciò implica che il racconto per immagini che proponiamo sia alquanto arbitrario e non abbia alcuna pretesa di ricostruire una cronologia degli eventi, di coprire le manifestazioni di piazza più importanti, o di proporre una panoramica esauriente dei materiali documentari a disposizione. Semplicemente, abbiamo cercato di descrivere una trasformazione delle forme di partecipazione collettiva alla vita politica nelle piazze bolognesi attraverso un repertorio di documenti (audio)visivi.

Il film si chiude col 1980 perché questa trasformazione si era ormai compiuta; semmai se ne stava inaugurando un'altra, che avrebbe mutato di nuovo le forme e i contenuti dell'uso pubblico della piazza, ma agendo almeno in parte nella stessa direzione, ovvero verso un rapporto sempre più critico tra società italiana e partiti di massa. Naturalmente, anche dopo il 1980 il Pci o le altre forze politiche - a Bologna come altrove - continuarono a organizzare manifestazioni, comizi, feste in piazza. $\mathrm{E}$ a ben vedere, certe adunate pubbliche presentavano ben poche differenze rispetto al passato (per esempio, le immagini dei funerali per la strage del 2 agosto 1980 restituiscono una piazza del tutto simile a quella di sei anni prima per la strage del treno Italicus, a partire dalla retorica antifascista e dallo sventolio delle bandiere rosse). Tuttavia, perlomeno nei fondi archivistici consultati presso Home Movies e l'Istituto Parri, le testimonianze filmiche della presenza dei partiti, e di quello comunista in particolare, nello scenario urbano degli anni Ottanta si fanno più rare. Come se nel frattempo fosse scomparsa non solo la vecchia "piazza rossa", ma anche l'attenzione dei cineamatori nei confronti della mobilitazione di massa nelle sue forme più tradizionali. Ma tutto sommato, anche questa latitanza è indizio di un mutamento del clima politico. 
